\section{Predictive Factors Of Appropriate Shock Therapy In Patients With Implantable Cardioverter Defibrillator}

\title{
Abstract
}

Appropriate shock therapy (AST) occurs in the minority of patients with implantable cardioverter defibrillators (ICDs). Evaluating predictors of ICD therapy may be helpful in developing risk stratification strategies for these patients. This could help the physician with his decision to offer ICD implantation. Expanding the indication to more permissive uses of ICD implantation seems to have resulted in selection of patients who are less likely to benefit from ICD. We assessed which patients received AST and whether there were any predictive factors.

Sahar Mouram ${ }^{1}$ MD. Mohamed Belhameche ${ }^{2}$ MD.

1 Cardiology B Departement, Faculty of medicine and pharmacy, Rabat.

2 Cardiology department, Jossigny hospital, Marne la vallée. Paris. France.

\section{Contact information:}

Sahar Mouram, Cardiology B department. Faculty of medicine and pharmacy, Rabat, Morrocco.

mouram.sahar@gmail.com

\section{Keywords}

Implantable cardioverter defibrillators, Shock therapy, risk factors.

\section{Introduction}

Automatic implantable cardioverter defibrillators (ICDs) are standard therapy for high-risk patients with life threatening ventricular arrhythmias. The ICD has been shown to be the optimal treatment for secondary prevention in patients with previous aborted sudden death or life threatening cardiac arrhythmias 1 and primary prevention in high risk patients with coronary artery disease and impaired left ventricular function 2,3. Previous studies have shown that only a minority of patients implanted with ICDs actually received appropriate shock therapy (AST). These tend to be sicker patients with impaired LV function. By the way there is limited information regarding the determinants of appro- priate ICD therapy in patients with reduced ventricular function. A history of a previous positive electrophysiology study is known to be a predictor of subsequent AST. We set out to determine the predictive factors of AST.

Electrical storm is an increasingly common and life-threatening syndrome that is defined by 3 or more sustained episodes of ventricular tachycardia, ventricular fibrillation, or appropriate shocks from an implantable cardioverter-defibrillator within 24 hours. The clinical presentation can be dramatic. Electrical storm can manifest itself during acute myocardial infarction and in patients who have structural heart disease, an implantable cardioverter-defibrillator, or an inherited arrhythmic syndrome. The presence or absence of structural heart disease 
and the electrocardiographic morphology of the presenting arrhythmia can provide important diagnostic clues into the mechanism of electrical storm. Electrical storm typically has a poor outcome.

The effective management of electrical storm requires an understanding of arrhythmia mechanisms, therapeutic options, device programming, and indications for radiofrequency catheter ablation. Initial management involves determining and correcting the underlying ischemia, electrolyte imbalances, or other causative factors. Amiodarone and $\beta$-blockers, especially propranolol, effectively resolve arrhythmias in most patients. Non pharmacologic treatment, including radiofrequency ablation, can control electrical storm in drug-refractory patients. Patients who have implantable cardioverter-defibrillators can present with multiple shocks and may require drug therapy and device reprogramming. After the acute phase of electrical storm, the treatment focus should shift toward maximizing heart-failure therapy, performing revascularization, and preventing subsequent ventricular arrhythmias.

There is pre procedural variables wich predict therapy delivery. These include left ventricular function, NHYA class and the results of programmed electrical stimulation ( PES) and other predictors factors.

\section{Effect of Ejection Fraction on Shock Occurrence and Survival}

Impaired LV function is a well-recognized predictor of AST. Grimm and al 4 in there series of two hundred forty one patients had demonstrated that Poor left ventricular function (ejection fraction inferior to $30 \%$ ) was associated with an earlier occurrence of both appropriate and any spontaneous ICD shocks $(p=0.001)$. Ejection fraction $<30 \%$ was the best predictor of both total arrhythmic death $(p=0.019)$ and total mortality $(p=0.003)$. These findings are similar to the results of Levine and colleagues 5 who found an ejection fraction $<25 \%$ predictive for earlier ICD discharge and shorter survival after ICD discharge. This confirm the finding of Kim and colleagues 6 that an ejection fraction $<30 \%$ is associated with a higher total arrhythmic and total mortality after ICD implant. Jagmmet et al 7 used secondary analysis in one arm of a multicenter randomized clinical trial in patients with a previous myocardial infarction and reduced left ventricular function ( MADIT-II study 719 Patients ) multiple hospitalization for heart failure - and New York Heart Association functional class superior to II were associated with increased risk for appropriate ICD therapy for ventricular tachycardy (VT) ventricular fibrillation (VF), or death. These results provide important mechanistic information, suggesting that worsening clinical condition and cardiac instability are subsequently associated with a significant increase in the risk for appropriate ICD therapy (for VT/VF) and death. This is the first study to highlight the importance of interim measures of clinical instability and their power to identify a subgroup (within this already high-risk population) at greater risk for ICD therapy and death. For Rinaldi and al 8 in their study of 155 patients. Those receiving AST had worse LV function with an approximate $10 \%$ decrease in EF (37.5\% vs. $47.8 \%$, $P$ inferior to 0.0001). Impaired LV function is a well-recognised predictor of AST.

\section{Programmed Electrical Stimulation (PES)}

In the study of Rinaldi and al 8, $59 \%$ of patients underwent PES at the time of ICD implant. He concluded that PES does not appear useful in predicting shock therapy in DCM patients and a negative PES should not preclude ICD implantation in this group.

PES is Known to predict further arrhythmia in hight risk patients and early studies reported PES induced arrhythmia in 9-20\% of post infarction patients with non sustained VT 9,10.In the MUSST study of 2202 patients undergoing PES 704 were classified as positive. Grimm and al 4 studied 241 ICD patients and found that VT at EPS was a predictor of AST.These findings were supported by those of Tebbenjohans 11. 
Andrews and al 12 studied patients with positive PES structural heart disease and unexplained syncope that were implanted with ICDs and found the ICD discharge rate was comparable to patients presenting with ventricular arrhythmias. In addition, appropriate and any spontaneous shocks occurred significantly later in patients who had only rapid polymorphic VT or VF induced before ICD implantation 4.

\section{High Risk Groups Dilated Cardiomyopathy}

Rinaldi and al 8 found a particular incidence of both appropriate and inappropriate therapy in their patients with dilated cardiomyopathy. The finding of an increased diastolic dimension is in keeping with the fact that patients with larger hearts and poorer LV function receive AST.In the study of Otmani and al dilated cardiomyopathy was one of the independent predictors of appropriate ICD 13. Ventricular arrhythmias are frequent in patients with DCM with $42-60 \%$ of patients showing at least non-sustained VT on Holter monitoring 14.

\section{Effect of Anti arrhythmic Drug Treatment}

For Grimm and al 4 in his study for 241 patients to determinate the effect of amiodarone.this effect was independently associated with later occurrence of any spontaneous shocks, but there was no significant difference in the occurrence of appropriate shocks in patients with and without amiodarone at implantation. This finding may merely reflect differences in patient selection for amiodarone therapy at implantation that were not accounted for by the clinical variables such as frequency and hemodynamic tolerance of the clinical VT and intolerance or failure of other agents. Treatment with class 1 anti arrhythmic drugs at implantation was associated with a higher total mortality but not with sudden death or total arrhythmic death. Of note, the lack of increased sudden death or total arrhythmic death rate in patients with class 1 agents at implantation excludes a causal relation between the increased total mortality and potential pro arrhythmic effects of class 1 agents. Differences in patient characteristics other than the variables may also account for the higher non arrhythmic mortality of patients with class 1 agents at implantation.

\section{Effect of the Clinical Presentation :}

Both appropriate and any spontaneous shocks occurred significantly later in patients who presented with a cardiac arrest before ICD implantation than in patients who presented with syncope or VT without syncope in the study of Grimm and al 4.This finding may be due to differences in the electrophysiological substrate in patients with cardiac arrest and only inducible polymorphic VT or VF as compared with patients with inducible uniform VT presenting with VT with or without syncope.

\section{Heart disease}

Similar to the results of Levine and colleagues 15, Reiter and al 16 found no difference in shock occurrence or survival curves among patients with coronary artery disease, non ischemic cardiomyopathy, valvular or congenital heart disease, and primary electrical disease. However, Zilo and al 17 found a higher incidence of shocks in patients with coronary disease. This difference may in part be due to differences in patient population as well as the lack of multivariate analysis in both studies.

\section{Age and Sex}

Similar to the results of Levine and coworkers Meyerburg and al 18 age and sex of patients in this study were not independently associated with shock occurrence or survival after ICD implantation.

\section{Cardiac risk factors}

For Desai and al 19 in his study of 549 patients with heart failure significant independent prognostic factors for appropriate ICD shocks were smoking (odds ratio 3.7) and statins (odds ratio 0.54). 
smoking significantly increased the incidence of appropriate ICD shocks 3.7 times, and the use of statins significantly reduced appropriate ICD shocks by $46 \%$.

In the MADIT II trial, smoking significantly increased the incidence of appropriate ICD shocks 2.1 times and inappropriate ICD shocks 2.9 times 20. In 209 patients with heart failure and combined cardiac resynchronization-ICD therapy, smoking significantly increased the incidence of appropriate ICD shocks by 3.5 times; The use of statins significantly reduced the incidence of appropriate ICD shocks by $54 \%, 21$ by $34 \%$ in MADIT \| 22 by $60 \%$ in 362 patients with coronary artery disease treated with an ICD for ventricular tachycardia/ventricular fibrillation 23 and from $57 \%$ to $22 \%$ in 78 patients with coronary artery disease treated with an ICD for life-threatening ventricular arrhythmias 24.

The potential mechanisms for statins reducing appropriate ICD shocks for ventricular tachycardia/ ventricular fibrillation could include their numerous beneficial pleiotropic effects, in addition to their favorable effects on serum lipids. By reducing high serum low-density lipoprotein cholesterol levels and increasing low serum high-density lipoprotein cholesterol levels, statins can slow the rate of progression of coronary atherosclerosis. Statins can decrease the inflammatory component of atherosclerosis progression, in which oxidized low-density lipoprotein cholesterol exerts an adverse effect on endothelial cells, smooth muscle cells, and macrophages.

\section{Effect of CRT on dispersion of repolarization}

The impact of cardiac resynchronization therapy (CRT) on dispersion of repolarization is controversial. The benefit of CRT on sudden cardiac death has been demonstrated only after 3 years follow-up.The purpose of the study of LeLouche and al 25 was to explore the immediate effect of CRT on dispersion of repolarization and to define the value of dispersion of repolarization parameters as predictors of appropriate implantable cardioverter-defibrillator (ICD) therapy. Data from 100 patients who underwent CRT-ICD placement were analyzed retrospectively.. ECG indices of dispersion of repolarization before and immediately after CRT implantation (QT dispersion, Tpeak-Tend [Tp-e], and Tp-e dispersion) were measured. In patients who were upgraded to a biventricular system, Tp-e did not increase significantly after CRT. However, Tp-e increased significantly after CRT in patients with left bundle branch block or narrow QRS at baseline. After 12-month follow-up, 22 patients had received appropriate ICD therapy. ICD therapy and no ICD therapy groups had similar baseline characteristics, such as secondary prevention and ischemic cardiomyopathy. Postimplantation Tp-e was the only independent predictor of future ICD therapy $(P=0.02)$. So, Immediately after CRT, Tp-e did not increase in patients who received a biventricular upgrade; however, Tp-e did increase in patients with preimplantation left bundle branch block or narrow QRS. Postimplantation Tp-e was the only independent predictor of appropriate ICD therapy.

\section{Serum markers}

Kanoupakis E,M and al 26 investigated prospectively whether serum markers of collagen turnover could be used as predictors for the occurrence of malignant ventricular arrhythmias in patients with nonischemic dilated cardiomyopathy (NIDC) who had received an implantable cardioverter-defibrillator (ICD) for primary prevention. Background Extracellular matrix alterations in NIDC might provide electrical heterogeneity, thus potentially contributing to the occurrence of ventricular arrhythmia and subsequent sudden cardiac death (SCD). Serum C-terminal propeptide of collagen type-I, C-terminal telopeptide of collagen type-I, matrix metalloproteinase (MMP)-1, and tissue inhibitor of MMP-1 were measured as markers of collagen synthesis and degradation in 70 patients with mild to moderate symptomatic heart failure due to NIDC with left 
ventricular ejection fraction 35\%, who received an ICD for primary prevention of SCD. Patients were evaluated for any appropriate ICD delivered therapy, whether shock or antitachycardia pacing, during a 1-year follow-up period. Appropriate device therapies were delivered in 14 of the 70 patients during the follow-up period, with antitachycardia pacing in 2, antitachycardia pacing with shocks in 4, and shocks in 8. Pre-implantation serum concentrations of C-terminal telopeptide of collagen type-I levels were significantly higher in patients who had appropriate ICD-delivered therapy than in those who did not have any therapy. The same was true for baseline MMP-1 and tissue inhibitor of MMP-1. As extracellular matrix alterations affect the arrhythmogenic substrate in NIDC, serum markers of collagen turnover could predict arrhythmic events in ICD recipients.

\section{Myocardial fibrosis}

Leah lles and al 27 evaluate the association between regional myocardial fibrosis and ventricular arrhythmias in patients with cardiomyopathy. He prospectively enrolled 103 patients meeting criteria for ICD implantation for primary prevention of SCD. Cardiac magnetic resonance imaging was performed before device implantation. Regional fibrosis was identified with late gadolinium enhancement (LGE). Median follow-up was 573 days. The LGE identified regional fibrosis in 31 of 61 (51\%) patients with nonischemic cardiomyopathy (NICM) and in all 42 patients with ischemic cardiomyopathy (ICM). There was a 29\% (9 of 31) discharge rate in the NICM group with LGE compared with a $14 \%$ (6 of 42) discharge rate in the ICM group (p:NS). There were no ICD discharges in the NICM group without LGE, which was significantly lower than the rate observed in both the ICM patients ( $p$ 0.04) and the NICM patients with LGE ( $p$ inferior to 0.01). Left ventricular ejection fraction was similar in patients with and without device therapy (24 12\% vs. 26 $8 \%$, p:NS) and those with or without LGE (25 9\% vs. 26 9\%, p :NS). Patients with advanced cardiomyopathy and myocardial fibrosis demonstrated by LGE on cardiac magnetic resonance imaging have a high likelihood of appropriate ICD therapy. Correspondingly, absence of LGE may indicate a lower risk for malignant ventricular arrhythmias.

\section{Depression :}

Whang and al 28 sought to examine the relationship between symptoms of depression and shock-treated ventricular arrhythmias among implantable cardioverter-defibrillator (ICD) patients. Depression predicts mortality in patients with coronary artery disease (CAD), but whether this is via an increased risk of fatal ventricular arrhythmias is unclear. He prospectively analyzed data on symptoms of depression and risk of ventricular arrhythmia (ventricular tachycardia/ventricular fibrillation [VT/VF]) resulting in ICD discharge in the Triggers of Ventricular Arrhythmias (TOVA) study. Among 645 patients with baseline assessments, 90 (14\%) were mildly depressed and 25 (3.9\%) were moderately to severely depressed. Moderate/severe depression was associated with time to first shock for VT/VF (hazard ratio [HR] 3.2, 95\% confidence interval [Cl] 1.1 to 9.9 ) and all shocks for VT/VF including recurrent episodes (HR 3.2, 95\% Cl 1.2 to 8.6). The risk of shock for VT/VF was associated with depression severity in the total population ( $p$ for trend 0.02) and among patients with CAD ( $p$ inferior to 0.01), even after controlling for multiple confounders. More severe symptoms of depression predict shocks for VT/ VF among ICD patients. The elevated risk of VT/VF among patients with CAD and depression suggests that arrhythmia may contribute significantly to total mortality in this subgroup.

\section{Other factors}

For Bhonsale and al 29 in his study of $84 \mathrm{~Pa}-$ tients With Arrhythmogenic Right Ventricular Dysplasia/Cardiomyopathy Undergoing Implantable Cardioverter-Defibrillator Implantation for Primary 
Prevention.Inducibility at electrophysiologic study ( $p$ inferior to 0.005), presence of non sustained ventricular tachycardia ( $p$ inferior to 0.001) and Holter premature ventricular complex count 1,000/24 h ( $p$ inferior to 0.024) were identified as significant predictors of appropriate ICD therapy.

\section{Conclusion}

Evaluating predictors of ICD therapy can be helpful in developing risk stratification strategies for patients. This could help the physician with his decision to offer ICD implantation.

\section{Conflict of interest}

None.

\section{References}

1. The antiarrhythmic versus implantable defibrillators (AVID) investigators. A comparison of antiarrhythmic drug therapy with implantable defibrillators in patients resuscitated from near-fatal ventricular arrhythmias. N Engl J Med 1997;337:1576-83.

2. Moss AJ, Hall WJ, Cannom DS.Improved survival with an implanted defibrillator in patients with coronary artery disease at high risk for ventricular arrhythmia.N Engl J Med, 335 (1996), pp. 1933-1940.

3. Buxton $A E$, Lee $K L$, Fisher JD.A randomised study of the prevention of sudden death in patients with coronary artery disease.N Engl J Med, 341 (1999), pp. 1882-1890.

4. Grimm W, Flores BT and Marchlinski FE. Shock Occurrence and Survival in 241 Patients With Implantable CardioverterDefibrillator Therapy. Circulation.1993;87(6): 1880-88.

5. Grunwald L,Guarnieri L, Aarons D, Griffith LSC : Predictors of first discharge and subsequent survival in patients with automatic implantable cardioverter-defibrillators. Circulation 1991;84: 558-566.

6. Kim SG, Fisher JD, Choue CW, Gross J, Roth J, Ferric KJ, Brodman $R$, Furman S: Influence of left ventricular function on outcome of patients treated with implantable defibrillators. Circulation
1992;85:1304-1310.

7. Jagmeet $P$, Hall WJ, McNitt $S$, Wang $H$, Daubert JP, Zareba W, Ruskin JN, Moss AJ, and the MADIT-II Investigators. Journal of the American College of Cardiology. 2005.46(9) : 1712-20.

8. Rinaldi CA, Simon RDB, Baszko A, Bostock J, Elliot D, Bucknall C, Jaswinder SG. Can we predict which patients with implantable cardioverter defibrillators receive appropriate shock therapy? A study of 155 patients International Journal of Cardiology.88(1) :69-75.

9. Denniss AR, Richards DA, Cody DV..Prognostic significance of ventricular tachycardia and fibrillation induced at programmed stimulation and delayed potentials detected on the signal averaged electrocardiograms of survivors of acute myocardial infarction. Circulation, 74 (1986), pp. 731-745

10. Roy D, Marchand E, Theroux P.Programmed ventricular stimulation in survivors of an acute myocardial infarction. Circulation, 72 (1985), pp. 487-494.

11. Tebbenjohanns J, Schumacher, Jung W.Predictors of outcome in patients with transvenous cardioverter defibrillatorsAm Heart J, 127 (1994), pp. 1086-1089.

12. Andrews NP, Fogel RI, Pelargonio G. Implantable defibrillator event rates in patients with unexplained syncope and inducible sustained ventricular tachyarrhythmias: a comparison with patients known to have sustained ventricular tachycardia.JACC, 34 (1999), pp. 2023-2330.

13. Otmani A, Trinquart $L$, Marijon E, Lavergne $T$, Waintraub $X$, Lepillier A, Chatellier G, Le Heuzey JY.On Behalf of the EVADEF investigators.Rates and predictors of appropriate implantable cardioverter-defibrillator therapy delivery: Results from the EVADEF cohort study. American Heart Journal 158(2).2009:23037.

14. Huang SK, Messer JV, Denes P. Significance of ventricular tachycardia in idiopathic dilated cardiomyopathy: observations in 35 patients Am J Cardiol, 51 (1983), pp. 507-512.

15. Levine JH, Mellits ED, Baumgardner RA, Veltri EP, Mower M, Grunwald L, Guarnieri T, Aarons D, Griffith LSC: Predictors of first discharge and subsequent survival in patients with automatic implantable cardioverter-defibrillators. Circulation 1991;84: 558-566

16. Reiter MJ, Fain ES, Senelli KM, Cadence Investigators: Determinants of recurrent ventricular arrhythmias in patients with implantable pacemaker/defibrillators. Circulation 1991;84(suppl II):II-426;

17. Gross J, Song SL, Buckingham T, Furman S, Bilitch Registry Group: Influence of clinical characteristics and shock occurrenceon ICD patient outcome: A multicenter report. PACE 1991;14: 18811886;

18. Myerburg RJ, Luceri RM, Thurer R, Cooper DK, Zaman L, Interian A, Fernandez P, Cox M, Glicksman F, Castellanos A: Time to first shock and clinical outcome in patients receiving an automatic implantable cardioverter-defibrillator. JAm Coll Cardiol 
1989;14:508-514

19. Desai H, Aronow WS, Ahn C, Gandhi K, Hussain S, Hoang ML, Sharma M, Frishman WH, Cohen M, Sorbera C, Risk Factors for Appropriate Cardioverter-Defibrillator Shocks, Inappropriate Cardioverter-Defibrillator Shocks, and Time to Mortality in 549 Patients With Heart Failure. The American Journal of Cardiology. 2010;105:1336-1338).

20. Goldenberg I, Moss AJ, McNitt S, Zareba W, Daubert JP, Hall WJ, Andrews ML; Multicenter Automatic Defibrillator Implantation Trial-II Investigators. Cigarette smoking and the risk of supraventricularand ventricular tachyarrhythmias in highrisk cardiac patients with implantable cardioverter-defibrillators. J Cardiovasc Electrophysiol2006;17:931-936.

21. Desai H, Aronow WS, Tsai F, Ahn C, Lai HM, Amin H, Gandhi K, Frishman WH, Cohen M, Sorbera C. Statins reduce appropriate cardioverter- defibrillator shocks and mortality in patients with heart failure and combined cardiac resynchronization and implantable cardioverter-defibrillator therapy. J Cardiovasc Pharmacol Ther 2009;14:176-179.

22. Vyas AK, Guo H, Moss AJ, Olshansky B, McNitt SA, Hall WJ, ZarebaW, Steinberg JS, Fischer A, Ruskin J, Andrews ML, for the MADIT-II Research Group. Reduction in ventricular tachyarrhythmias with statins in the Multicenter Automatic Defibrillator Implantation trial (MADIT)-II. J Am Coll Cardiol 2006;47:769 -773.

23. Mitchell LB, Powell JL, Gillis AM, Kehl V, Hallstrom AP; AVID Investigators. Are lipid-lowering drugs also antiarrhythmic drugs? An analysis of the antiarrhythmics versus implantable defibrillators (AVID) trial. J Am Coll Cardiol 2003;42:81-87.

24. De Sutter J, Tavernier R, De Buyzere M, Jordaens L, De Backer G. Lipid lowering drugs and recurrences of life-threatening ventricular arrhythmias in high-risk patients. J Am Coll Cardiol 2000;36:766-772

25. Lellouche N,De Diego G, Akopyan G, Boyle GB, Mahajan, Cesario DA, Wiener I, Shivkumar K.Changes and predictive value of dispersion o.f repolarization parameters for appropriate therapy in patients with biventricular implantable cardioverterdefibrillators. Heart rythme. 4(10).2007:1274-83.

26. Kanoupakis EM . Manios EG, Kallergis EM, Mavrakis HE, Goudis CA, Saloustros IG .Milathianaki ME,Chlouverakis GI, Vardas PE. Serum Markers of Collagen Turnover Predict Future Shocks in Implantable Cardioverter-Defibrillator Recipients With Dilated Cardiomyopathy on Optimal Treatment. Journal of the American College of Cardiology .55(24). 2010 :2753-9.

27. Leah I, Pfluger H, Lefkovits L, Butler MJ, Kistler PM, Kaye DM, Taylor AJ. Myocardial Fibrosis Predicts Appropriate Device Therapy in Patients With Implantable Cardioverter-Defibrillators for Primary Prevention of Sudden Cardiac Death. Journal of the American College of Cardiology. 57(7) $2011: 821-8$
28. Whang W. Albert CM, Sears SM, Lampert R, Conti JB, Wang PG, Jagmeet PS. Ruskin JN, Muller JE, Mittleman MA, TOVA Study Investigators ;Depression as a Predictor for Appropriate Shocks Among Patients With Implantable Cardioverter-Defibrillators. Journal of the American College of Cardiology. (45) 7. 2005 1090-5.

29. Bhonsale A., James CA, Tichnell C, Murray B, Gagarin D, Binu P, Darshan D, MD, Tedford R,

30. Russell SD, Abraham T, Tandri H, Judge DP, H Calkins. Incidence and Predictors of Implantable Cardioverter-Defibrillator Therapy in Patients With Arrhythmogenic Right Ventricular Dysplasia/ Cardiomyopathy Undergoing Implantable CardioverterDefibrillator Implantation for Primary Prevention. Journal of the American College of Cardiology. 58(14).2011:1485-96.

\section{Comment on this article:}

\section{$4[$ in $8+\mathbf{S} P$}

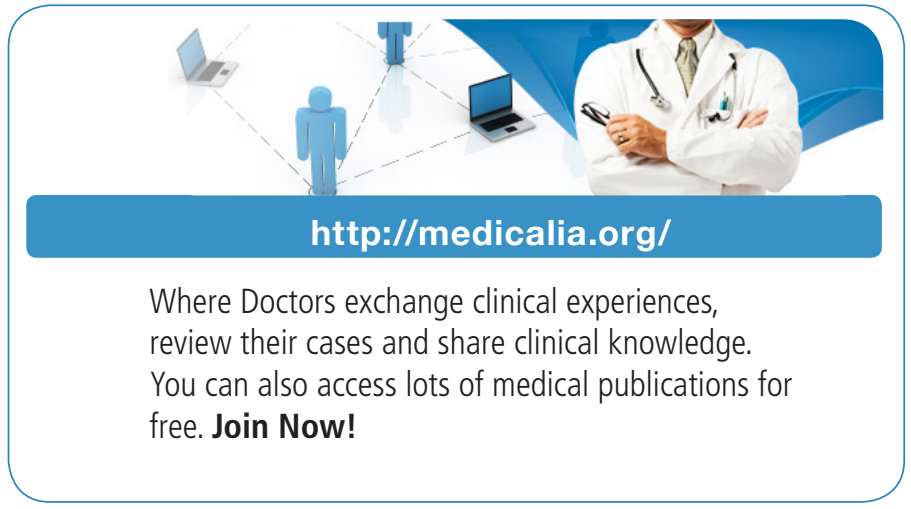

\section{Publish with iMedPub}

\section{http://www.imed.pub}

International Archives of Medicine is an open access journal publishing articles encompassing all aspects of medical science and clinical practice. IAM is considered a megajournal with independent sections on all areas of medicine. IAM is a really international journal with authors and board members from all around the world. The journal is widely indexed and classified Q1 in category Medicine. 